\title{
Tracción de la base de la lengua mediante fijación para el tratamiento de la apnea del sueño
}

\author{
J.L. Cebrián Carretero1, J.M. Muñoz Caro², M. Chamorro Pons³, L García Arana3, \\ M.J. Morán Soto ${ }^{1}$, M. Burgueño García ${ }^{1}$
}

\begin{abstract}
Resumen: El síndrome de apnea obstructiva del sueño (SAOS) constituye en la actualidad un problema de salud importante, tanto por su prevalencia como por la morbilidad que provoca sobre el aparato cardiovascular y la calidad de vida del paciente. Actualmente, la base del tratamiento es la administración de presión positiva continua durante el sueño (CPAP) que, si bien tiene una elevada eficacia, no es tolerada en muchos casos. Los tratamientos quirúrgicos han sido variados. En un principio se centraron en el sistema velofaríngeo con mejorías no superiores al $50 \%$ a largo plazo. En nuestro planteamiento terapéutico identificamos, mediante pruebas funcionales y de imagen, las regiones implicadas en cada caso. Entre ellas destaca la base de la lengua. La técnica que presentamos constituye una modificación a las ya descritas para el avance de la base lingual. Consiste en una fijación controlada de la misma a la cortical vestibular de la mandíbula con un punto de hilo de monofilamento pasado a través de dos orificios, sin necesidad de aditamentos especiales. Conseguimos una estabilización permanente con buenos resultados clínicos y polisomnográficos a largo plazo. Las complicaciones estuvieron en relación con problemas deglutorios en el postoperatorio inmediato.
\end{abstract}

Palabras clave: SAOS; CPAP; Base de lengua; Faringoplastia.

\begin{abstract}
Sleep apnoea síndrome constitutes an important health problem because of its prevalence and impact over the cardiovascular system and quality of life of the patient. The classic conservative treatment remains to be the CPAP. Although it has a high efficacy, it is not well tolerated by the patient in many cases. The surgical treatments were initially devoted to the velopharyngeal complex, being definitive in only $50 \%$ of the cases. Our therapeutical approach consists on a thorough examination of the different areas affected in every case, one of which is the tongue base. The surgical technique we present constitutes a modification of others already described to advance the tongue base. It gets a controlled fixation of the tongue to the vestibular cortex with a monofilament suture through two holes without any special instruments. We obtain a permanent stabilization with good long term results clinically and polysomnographically. The complications found were relationed with deglution problems in the inmediate posoperative period.
\end{abstract}

Key words: OSAS; CPAP; Tongue base; Pharyngoplasty.

1 Licenciado en Medicina y Cirugía. Especialista en Cirugía Oral y Maxilofacial. F.E.A.

2 Doctor en Medicina y Cirugía. Especialista en Cirugía Maxilofacial. F.E.A.

3. Licenciado en Medicina y Cirugía. Especialista en Cirugía Maxilofacial. Residente

Servicio de Cirugía Oral y Maxilofacial. Hospital Universitario La Paz, Madrid. España

Correspondencia:

José Luis Cebrián Carretero

Servicio de Cirugía Maxilofacial H.U. "La Paz"

Paseo de la Castellana, 261

28046 Madrid, España

\section{Introducción}

El síndrome de apnea obstructiva del sueño (SAOS) es un trastorno caracterizado por la aparición de pausas respiratorias durante el sueño por colapso de la vía aérea superior, que repercuten básicamente sobre el sistema nervioso y el aparato cardiorrespiratorio.

La primera referencia a este síndrome aparece unos trescientos años antes de Jesucristo, en la antigua ciudad griega de Heracleia, donde encontramos la descripción de Dionisio, uno de sus gobernantes, como un individuo «de en orme obesidad con dificultades para 
respirar, quedándose dormido fácilmente. Su médico ideó un artilugio con agujas largas y finas que se le clavaban en la piel con objeto de mantenerle despierto, ...». En el año 1871, el célebre Charles Dickens describe en su libro «Posthumous Papers of the Pickwick Club»a un criado llamado Joe, con gran sobrepeso, cara enrojecida y que se quedaba dormido fácilmente. Desde estas primeras descripciones hasta nuestros días el SAOS ha recibido múltiples denominaciones, y muchos son los avances hechos en el conocimiento de la enfermedad. En 1918, Sir William Osler calificó a los pacientes obesos con hipersomnia como «pickwician» dada la similitud encontrada con el personaje de Dickens, siendo Burwell quien en 1956 agrupó a estos pacientes en lo que llamó síndrome de Pickwick. En 1972, Christian Guilleminault acuñó el término síndrome de apnea obstructiva de sueño»para englobar a estos pacientes con obesidad, hipersomnia y anormal patrón respiratorio durante el sueño, y lo presentó ese mismo año en el primer simposio sobre patología respiratoria relacionada con el sueño, que se celebró en Italia organizado por Sadoul y Lugaresi. ${ }^{1}$ Allí nació el actual concepto de lo que hoy seguimos conociendo con el nombre de síndrome de apnea obstructiva del sueño.

Hasta la década de los 80, el único tratamiento existente para el SAOS era la traqueostomía. Hoy existen muchas opciones terapéuticas, aunque desgraciadamente ninguna totalmente satisfactoria. Entre las conservadoras destaca la CPAP (Continuous Positive Airway Pressure), ${ }^{2}$ mientras que entre las intervencionistas, las técnicas quirúrgicas son muy variadas. Hasta hace unos años, la mayoría trataban de modificar fundamentalmente la región velofaríngea, pues se basaban en la idea central de que el exceso de tejido blando palatino es el principal responsable de la génesis de apneas durante el sueño. Sin embargo, en los últimos años, los conceptos han cambiado, ya que se ha comprobado que en el SAOS se ven implicadas otras zonas de la vía aérea superior (hipertrofia adenoideo-amigdalar, base de la lengua, paredes faríngeas, etc.), y no sólo la región del paladar blando.3-5 Así, la identificación preoperatoria de la zona donde se produce la obstrucción ha ayudado a seleccionar a los candidatos para la úvulopalatofaringoplastia (UPFP), pudiendo ofrecer tratamientos quirúrgicos alternativos a los pacientes que presentan obstrucción a otros niveles, como septoplastias, descongestión submucosa de los cometes, láser sobre la base de la lengua, avances del músculo geniogloso o suspensión hioidea entre otros. ${ }^{4,6,7}$

En el presente trabajo describimos una técnica quirúrgica destinada para tratar a aquellos pacientes en los que la obstrucción al paso del aire se sitúa fundamentalmente en la base de la lengua y que puede ser combinada con otras técnicas en el tratamiento de pacientes con SAOS.

\section{Técnica quirúrgica}

La técnica del punto de tracción de la base de la lengua se realiza bajo anestesia general e intubación nasotraqueal. El primer paso consiste en realizar una incisión horizontal en el fondo del vestíbulo labial mandibular. Una vez incidido, se profundiza hasta el perióstio, que se separa del hueso subyacente. En este momento se practican dos trépanos bicorticales a ambos lados de la sínfisis. Estos trépanos deben trazarse de abajo a arriba y de medial a lateral, de manera que nos permitan pasar a través de ellos una lezna para alcanzar la base lingual. Para llevar a cabo esta maniobra, la lezna (montada con un hilo de monofilamento del 0) se introduce por uno de los orificios, mientras que con la otra mano se palpa la base lingual por detrás de la $\mathrm{V}$ del mismo lado con el fin de guíar el paso del instrumento. Una vez pasado, el ayudante desmonta el hilo de la lezna, y lo monta en una aguja cilíndrica viuda del tamaño suficiente para pasar de lado a lado la base lingual. En este momento la lezna se introduce por el otro orificio hacia la base lingual homolateral mediante la misma maniobra que empleamos en el otro lado, pero portando un hilo de alambre (Fig. 1). En este momento el ayudante toma el hilo de alambre sin desmontarlo de la lezna y le anuda el hilo de monofilamento El paso final consiste en traccionar de la lezna arrastrando los hilos de alambre y monofilamento, para terminar anudando éste último sobre sí mismo en la sinfisis mandibular (Figs. 2 y 3). Al mismo tiempo, el ayudante comprobará manualmente cómo se tensa y desplaza hacia delante la base lingual. Esta técnica se puede combinar con otras como la uvulopalatofaringoplastia en el caso de que exista obstrucción a otros niveles. El paciente debe ser derivado desde el quirófano a una unidad de reanimación postoperatoria 0 a una unidad de cuidados intensivos durante al menos 24 horas. Del mismo modo debe emplearse una sonda nasogástrica para alimentación durante los primeros días del postoperatorio, en los que la deglución se encuentra comprometida.

\section{Discusión}

El síndrome de apnea obstructiva del sueño (SAOS) es una entidad muy frecuente que padecen aproximadamente el $2-4 \%$ de los varones y $1-2 \%$ de las mujeres entre 40 y 60 años y que clínicamente se define por la presencia de un número excesivo de episodios de apnea-hipoapnea durante el sueño.

La consecuencia clínica más frecuente es la hipersomnia diurna crónica debida a la fragmentación del sueño, que supone un elevado coste social en términos de bajo rendimiento laboral y accidentes. Además, se han publicado estudios que demuestran la relación 


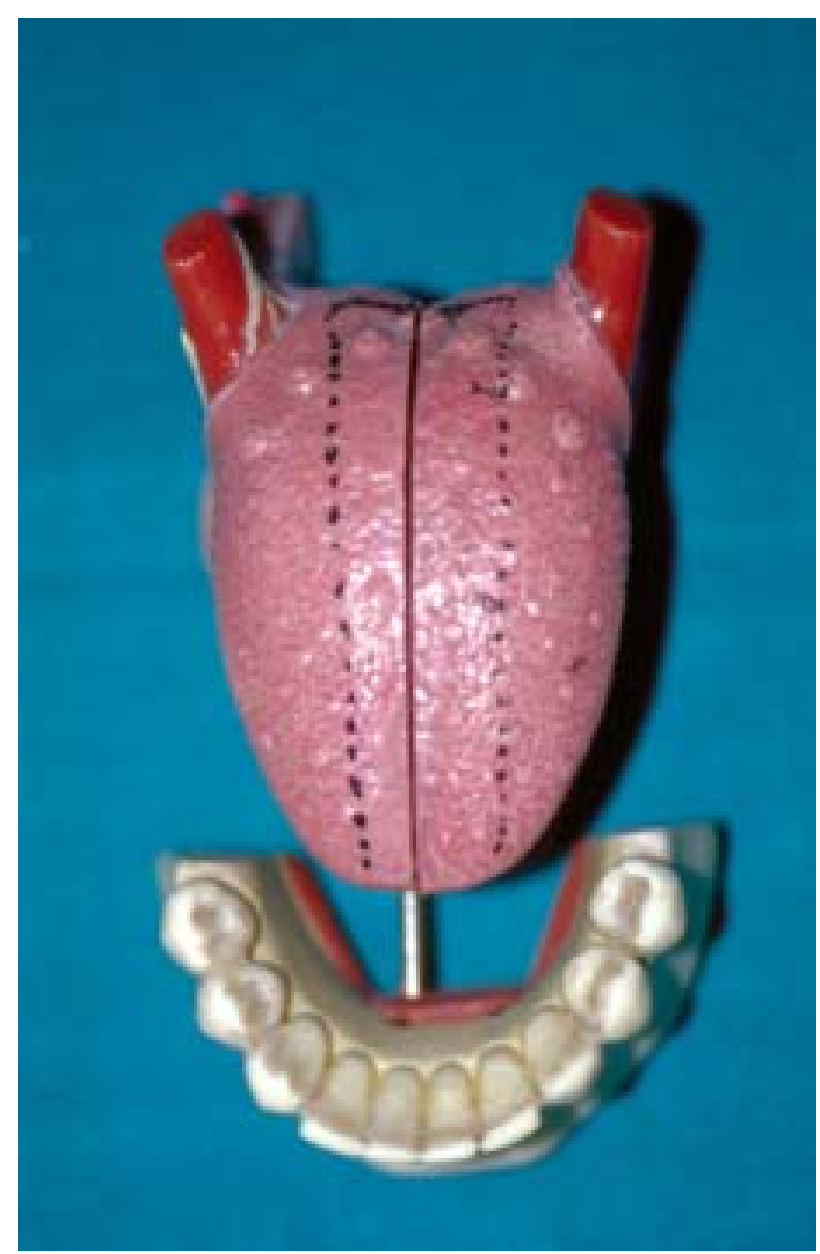

Figura 1. Trazado del camino que sigue el hilo de monofilamento en el espesor lingual.

existente entre el SAOS y enfermedades cardiovasculares tan importantes como la hipertensión arterial, cardiopatía isquémica, accidentes cerebrovasculares, hipertensión pulmonar, arritmias cardíacas, fallo cardíaco derecho e incluso disfunción ventricular, izquierda que pueden acabar con la vida del paciente. ${ }^{8}$

Este cuadro clínico se explica a través de una serie de cambios hormonales y metabólicos (déficit de hormona del crecimiento, depresión del sistema reninaangiotensina-aldosterona, elevación del péptido auricular natriurético, descenso de la testosterona, etc.) cuyo mecanismo fisiopatogénico exacto no es del todo conocido.

La combinación de la elevada prevalencia del SAOS con la amplia gama de consecuencias clínicas y sociales que conlleva, hacen absolutamente necesario llevar a cabo un diagnóstico precoz y un tratamiento adecuado para estos pacientes.

El diagnóstico de sospecha lo ofrece la clínica, pero para llegar a un diagnóstico de certeza, es necesario realizar una prueba de sueño (polisomnografía), en la que se demuestre un índice de apneas (pausas respiratorias $>10$ segundos) e hipoapneas (disminución de la saturación de oxígeno por debajo del 50\% durante

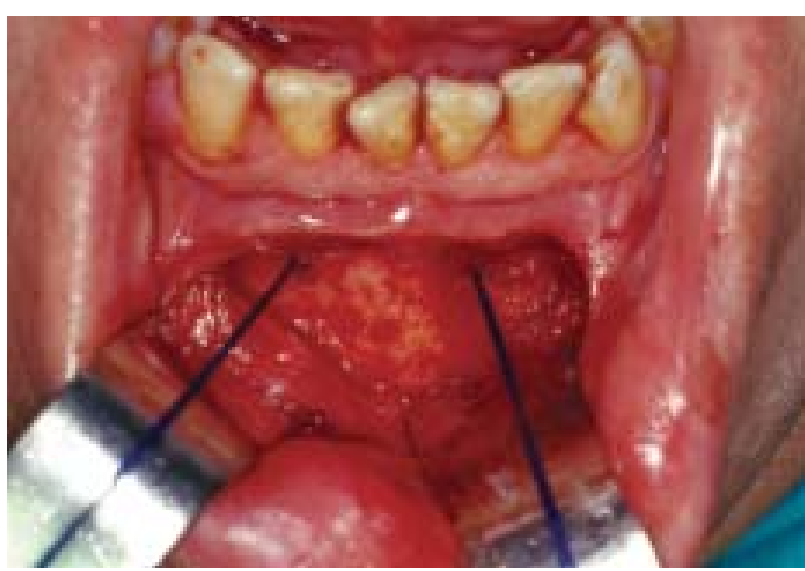

Figura 2. Posición de ambos cabos del hilo, emergiendo por los trépanos de la sínfisis mandibular, en el momento previo al anudado de ambos.

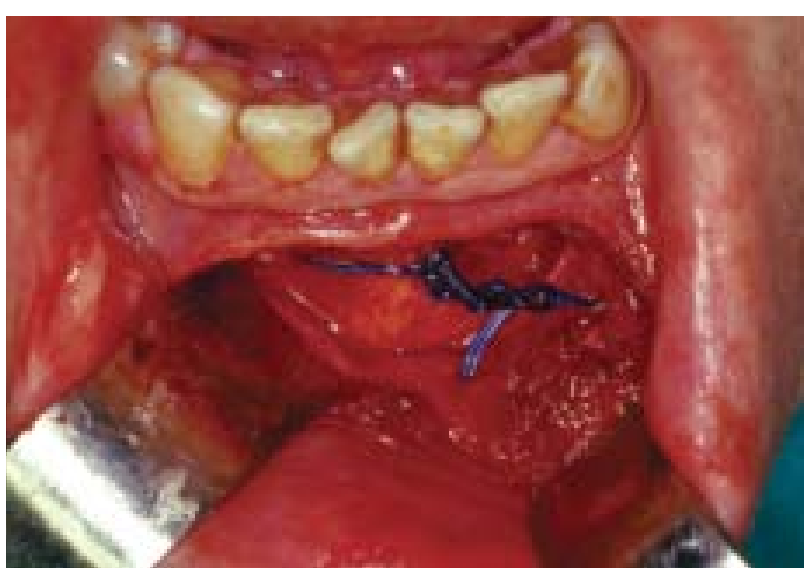

Figura 3. Situación definitiva del hilo tras realizar la sutura, tracción y anudado.

más de 10 segundos) superior a 10 por hora de sueño. Además, para distinguir entre apnea central y obstructiva, será necesario contar con pruebas de imagen, tanto estáticas (TC) como dinámicas (nasofibroscopia, vídeofluoroscopia), que pongan de manifiesto el lugar de la obstrucción.

Hasta la década de los 80, el único tratamiento existente para el SAOS era la traqueostomía permanente, sin embargo, hoy en día existen muchas opciones terapéuticas, aunque desgraciadamente ninguna totalmente satisfactoria. Entre las conservadoras destaca la CPAP (Continuous Positive Airway Pressure), descubierta en 1981 por Sullivan, ${ }^{2}$ que estableció la eficacia de la presión positiva continua nasal para eliminar los episodios de apnea. De esta manera la CPAP pasó a ser ampliamente reconocida y aceptada como tratamiento a largo plazo del SAOS. Sin embargo, la CPAP tiene grandes problemas de tolerancia a largo plazo, pues supone la dependencia crónica de una máquina que únicamente produce una mejoría sintomática sin curar la enfermedad. De esta forma, su tasa de utilización óptima (siete 


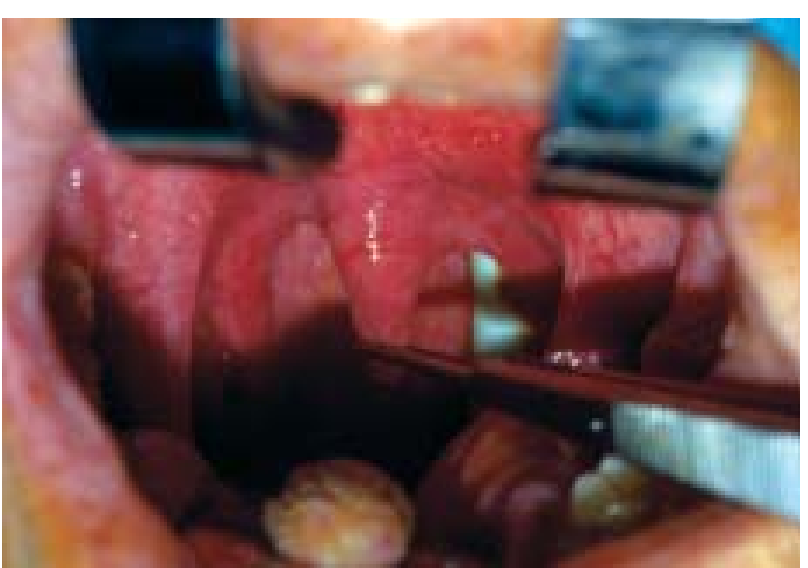

Figura 4. Paladar blando y úvula redundante en paciente afectado de SAOS.

horas por noche el $70 \%$ de las noches) es tan solo un $5,7 \%$.

Las diferentes técnicas quirúrgicas descritas para el tratamiento del SAOS tratan de producir cambios permanentes que solucionen los episodios de apnea. En un principio, la mayoría de ellas se centraron en la modificación de la región velofaríngea, basándose en la idea de que el exceso de tejido blando palatino es el principal responsable de la génesis de las apneas durante el sueño (Fig. 4). Así, en 1981 Fujita, ${ }^{9}$ demostró que la UPFP, técnica utilizada hasta entonces para el control de los ronquidos, reducía el número de apneas durante el sueño (Fig. 5). La mejoría clínica de los pacientes que se conseguía con etas técnicas motivó el auge por la cirugía de los tejidos blandos de la vía aérea superior como tratamiento del SAOS. Sin embargo, la cirugía velofaríngea por sí sola tiene un efecto limitado en el tratamiento del SAOS, presentando una efectividad que no supera el 50\%. ${ }^{10,11}$ Los resultados a largo plazo parecen demostrar un empeoramiento tanto subjetivo como del estudio polisomnográfico a los cinco años de la UPFP. Las causas de la dificultad que tiene este tipo de cirugía para resolver definitivamente el SAOS no están del todo claras, pero parece que se debe en cierta medida a que no se resuelve definitivamente la obstrucción a nivel palatino y, fundamentalmente, a que en muchos casos de SAOS se ven implicadas otras zonas de la vía aérea superior (hipertrofia adenoideoamigdalar, base de la lengua, paredes faríngeas, etc.), y no sólo la región del paladar blando.

Hoy en día, además del diagnóstico del síndrome mediante polisomnografía, se considera necesario el diagnóstico etiológico para la identificación preoperatoria de la zona donde se produce la obstrucción. Estos dos conceptos - diagnóstico y cirugía del agente causal- han revolucinado el tratamiento del SAOS y han permitido por primera vez ofrecer resultados predecibles y a largo plazo. ${ }^{6}$

En nuestro protocolo de diagnóstico, además de la

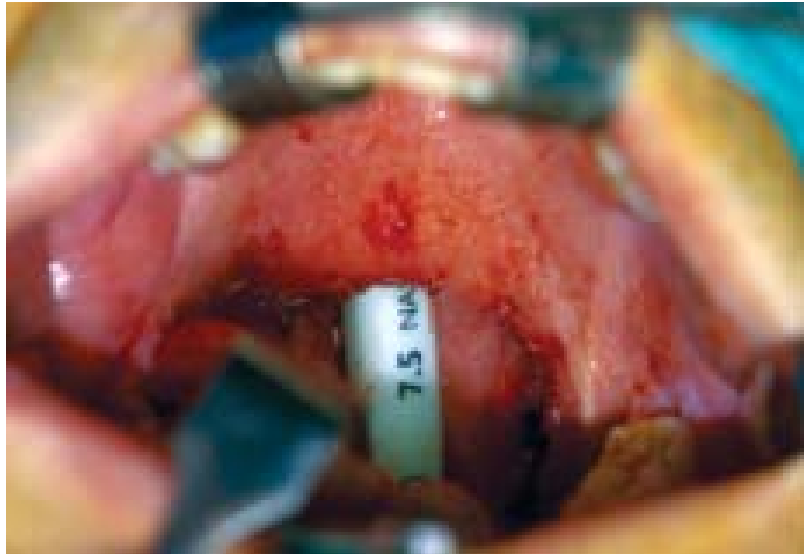

Figura 5. Aspecto del paladar tras realizar la úvulopalatofaringoplastia. Debe quedar suficiente tejido para evitar la incompetencia velofaríngea.

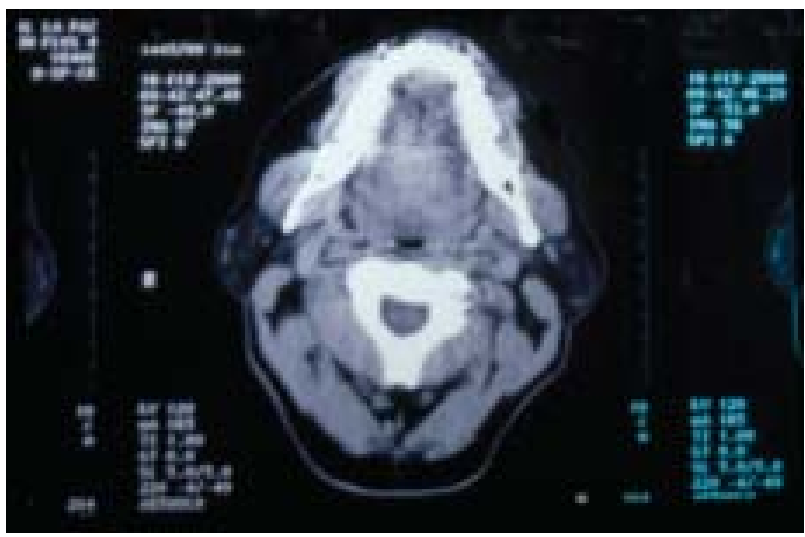

Figura 6. Tomografía computarizada de paciente con SAOS en la que se aprecia la importante obstrucción de la vía aérea en la región de la base de la lengua.

polisomnografía que demuestre el síndrome, incluímos un estudio cefalométrico, una nasofibroscopia y un scanner con cortes axiales de la vía aérea superior (Fig. 6).

Basándonos en estos estudios aplicamos una cirugía por etapas, en las que vamos tratando y solucionando progresivamente los diferentes puntos en los que encontramos dificultad al paso del aire. De este modo nos fijamos primero en el septo nasal y los cornetes, y en los casos indicados realizamos septoplastias y turbinectomías; luego, en la zona velofaríngea, y en los casos que lo precisan realizamos uvulopalatofaringoplastias; y finalmente en la región de la base de la lengua, donde realizamos la técnica de tracción lingual previamente descrita. A lo largo de este proceso tratamos de identificar malformaciones dentofaciales, p ej. retrusión mandibular. ${ }^{12,13}$

En cualquier caso, independientemente de las técnicas quirúrgicas empleadas, se debe instar al paciente a que abandone hábitos de riesgo (tabaco, abuso de alcohol, etc.) y por supuesto conseguir que disminuya de peso para mantener el índice de masa corporal por debajo de $35 .{ }^{14}$ 
El tratamiento de la región de la base de la lengua como zona de obstrucción al paso del aire ha cobrado un gran auge en los últimos años, pues se ha visto que se trata de una zona frecuentemente implicada en los casos de SAOS.15

La primera referencia a las suturas de tracción de la base lingual la encontramos en la reunión de la AAO - HNSF (Academia Americana de ORL) en San Diego en 1998, donde De Rowe y cols., ${ }^{16}$ presentaron la técnica en 35 pacientes que mejoraron o se curaron de su sintomatología. Posteriormente, la técnica fue reevaluada por Coleman, ${ }^{17}$ que la consideró como una opción prometedora. Las últimas referencias en la literatura corresponden de nuevo a los diseñadores de la técnica, que revisaron sus casos a medio plazo, considerando que constituía un método eficaz y seguro, si bien la mejoría era más subjetiva que en parámetros medibles durante la polisomnografía. En estos mismos artículos señalan como complicaciones más frecuentes la sialoadenitis y las alteraciones gastrointestinales derivadas de los problemas deglutorios y el sangrado de la base de la lengua. ${ }^{18}$

El método de fijación aquí presentado difiere en cierta medida del descrito y empleado en un principio por De Rowe y cols. ${ }^{16}$ Su sistema se basaba en un ancla oradada en su parte distal, a través del cuyo orificio se pasaba una sutura para relizar la fijación de la base lingual. El ancla se fijaba mediante un dispositivo angulado a la cortical interna mandibular. ${ }^{19}$

Las ventajas de nuestro método consiste en la obtención de una fijación más rígida y controlada al suturar en la regón vestibular y no en la lingual así como la posibilidad de aplicar la técnica sin necesidad de ningún dispositivo especial.

En nuestro Servicio hemos empleado la técnica en cuatro pacientes roncadores diagnosticados de apnea del sueño mediante estudio polisomnográfico y tratados durante años con CPAP, muy mal tolerada. Debido a la poca colaboración de los profesionales que realizan el tratamiento médico de estos pacientes en nuestro hospitqal, el reclutamiento fue al azar en pacientes que acudieron a nuestra consulta por otro problema y que declararon en sus antecedentes personales padecer SAOS.

En todos los pacientes se realizó la técnica de suspensión de la base lingual asociada a úvulopalatofaringoplastia. El postoperatorio cursó sin complicaciones, si bien resultó bastante incómodo por la odinofagia y las alteraciones de la deglución que desaparecieron aproximadamente dos semanas tras la cirugía. Tras un periodo de seguimiento de 3 a18 meses, los pacientes permanecen asintomáticos, sin ronquido y sin necesidad de emplear la CPAP. Durante este periodo a uno de los paciente se le realizó una septoplastia como parte del tratamiento de cirugía por etapas.
Aunque los resultados parecen prometedores no pueden ser considerados como defrinitivos y necesitamos seguir a más pacientes y durante más tiempo.

\section{Conclusiones}

No obstante podemos extraer dos conclusiones. La primera es que la cirugía es una alternativa válida a la CPAP y que puede ser curativa en muchas ocasiones; y la segunda es que dicha cirugía debe centrarse en los problemas anatómicos que causan la obstrucción. En este sentido, en los casos en que la obstrucción se encuentre en la base de la lengua la técnica de suspensión mandibular parece bastante prometedora. Una alternativa a esta técnica que se está desarrollando en los últimos años es la reducción del volumen de la base de la lengua mediante bisturí armónico. ${ }^{20}$

\section{Bibliografía}

1. Guilleminault C, Eldridge FL, Dement WC. Insomnia with sleep apnea: a new syndrome. Science 1973;181:856-8.

2. Sullivan CE, Issa FG, Berthon-Jones M, Eves L. Reversal of obstructive sleep apnea by continuous positive airway pressure applied through the nares. Lancet 1981;1:862- 5.

3. Biurrun Unzué O. Surgical treatment of obstructive sleep apnea syndrome: From palatopharyngeal surgery to multilevel surgical reconstruction of the pharynx. Basis, results and future perspectives. Acta Otorrinolaringol Esp 2001;52:578-90.

4. Biurrún O, Morello A. Traserra J. Surgical multiple level reconstruction of the upper airway for treatment of obstructive sleep apnea. Two clinical cases. Acta Otorrinolaringol Esp 1996;47:479-83.

5. Lu SJ, Chang SY, Shiao GM. Comparison between short term and long term postoperative evaluation of sleep apnoea after uvulopalatopharyngoplasty. J Laryngol Otol 1995;109:308-12.

6. Vilaseca I, Morello A, Montserrat JM, Santamaría J, Iranzo A. Usefulness of uvulopalatopharyngoplasty with genioglossus and hyoid advancement in the treatment of obstructive sleep apnea. Arch Otolaryngol Head Neck Surg 2002;128:435-440.

7. Sandler NA, Braun TW. Hyoid myotomy and suspension for obstructive sleep apnea syndrome. J Oral Maxillofac Surg 1997;55:656.

8. Haapaniemi JJ, Laurikanien EA, Halme P, Autila J. Long term results of tracheostomy for severe sleep apnea syndrome. ORLJ Otorrhinolaryngol Relat Spec 2001;63:13136.

9. Fujita S, Conway W, Zorick F, Roth T. Surgical correction of anatomic abnormalities in obstructive sleep apne syndrome: uvulopalatopharyngoplasty. Otolaryngol Head Neck Surg 1981;89:923-34.

10. Walker RP, Garutz T, Gopalsami C. Early polysomno- 
graphy findings and long-term subjective results in sleep apnea patients treated with laser-assisted uvuloplasty. Laryngoscope 1999;109:1438-41.

11. Walker RP, Gripp-Damberger MM, Gopalsami C. Uvulopalatopharyngoplasty versus laser-assisted uvuloplasty for the treatment of obstructive sleep apnea. Laryngoscope 1997;107:76-82.

12. Li KK, Powell NB, Riley RW, Guilleminault C. Distraction osteogenesis in adult obstructive sleep apnea syndrome: a preliminary report. J Oral Maxillofac Surg 2002;60:6- 10.

13. Nimyork Y, Mils PG, Waite PP. Maxillomandibular advancement surgery in obstructive sleep apnea syndrome patients: long-term surgical stability. J Oral Maxillofac Surg 1995;53:1414-17.

14. Ulnick KM, Debo RF. Postoperative treatment of the patients with obstructive sleep apnea. Otolaryngol Head Neck Surg 2000;122:233-6.

15. De Rowe A, Gunther E, Fibbi A, Lehtimaki K, Vahatalo $\mathrm{K}$, Maurer J, Ophir D. Tongue base suspension with a soft tissue-to-bone anchor for obstructive sleep apnea: preliminary clinical results of a new minimally invasive technique. Otolaryngol Head Neck Surg 2000;122:100-3.

16. De Rowe, Ophir D, Fabbi A, Günther E, Lehtimäki K, Maurer J, Vähätalo K. Tongue base suspension for sleep disorderer breathing. A new technique. Submitted to AAOHNSF 1998 Annual Meeting. San Antonio, Texas.

17. Coleman J, Bick PA. Suspension sutures for the treatment of obstructive sleep apnea and snoring. Otolaryngol Clin North Am 1999;32:277-85.

18. Woodson BT, De Rowe A, Hawke M, Wenig B, Ross EB Jr, Katsantonis GP, Mickelson SA, Bonham RE, Benbadis S. Otolaryngol Head Neck Surg 2000;122:395-401.

19. Woodson BT. A tongue suspension suture for obstructive sleep apnea and snorers. Otolaryngol Head Neck Surg 2001;124:297-303.

20. Woodson BT, Nelson L, Mickelson S, Huntley T, Sher A. A multiinstitutionalstudy of radiofrquency volumetric tissue reduction for OSAS. Otolaryngol Head Neck Surg 2001;125:303-11. 